\title{
Erratum to: Crime Places in Context: An Illustration of the Multilevel Nature of Hot Spot Development
}

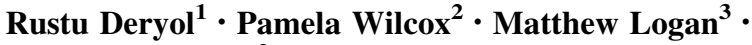 \\ John Wooldredge ${ }^{2}$
}

\section{Erratum to: J Quant Criminol DOI 10.1007/s10940-015-9278-1}

There is one correction to our original article. Table 3 was not displayed properly. All statistics presented were correct, but an incorrect variable name was provided with respect to the cross-level interaction term, and this might confuse readers. A corrected version of Table 3 is published with this erratum.

The online version of the original article can be found under doi:10.1007/s10940-015-9278-1.

\section{Rustu Deryol}

deryolr@sar.usf.edu

1 Department of Criminology, College of Arts and Sciences, University of South Florida-Sarasota Manatee, 8350 N. Tamiami Trail, Sarasota, FL 34243, USA

2 School of Criminal Justice, University of Cincinnati, Cincinnati, OH, USA

3 Department of Criminal Justice, California State University—San Bernardino, San Bernardino, CA, USA 
Table 3 Poisson hierarchical regression analysis results with level-2 predictors and cross-level interaction

\begin{tabular}{|c|c|c|c|c|}
\hline \multirow[t]{2}{*}{ Fixed effect } & \multicolumn{2}{|l|}{ Model 1} & \multicolumn{2}{|l|}{ Model 2} \\
\hline & Coeff. & SE & Coeff. & SE \\
\hline \multicolumn{5}{|l|}{ Block group mean crime } \\
\hline Base & $0.594^{*}$ & 0.126 & $0.558 *$ & 0.127 \\
\hline Commercial density & $0.438^{*}$ & 0.217 & $0.522 *$ & 0.228 \\
\hline Disadvantage & $0.092 *$ & 0.039 & $0.091 *$ & 0.038 \\
\hline Instability & 0.01 & 0.022 & 0.020 & 0.021 \\
\hline Felon residence & -0.017 & 0.012 & -0.017 & 0.012 \\
\hline Spatial lag & 0.015 & 0.037 & 0.011 & 0.039 \\
\hline \multicolumn{5}{|c|}{ Carry-out liquor $\times$ on-premises drinking $\times$ bus routes } \\
\hline Base & $-0.454 *$ & 0.061 & -0.008 & 0.205 \\
\hline $\begin{array}{l}\text { Commercial density } \\
\quad \text { (cross-level interaction) }\end{array}$ & - & - & $-1.076^{+}$ & 0.57 \\
\hline Random effect & Var (SD) & Chi sq. & Var (SD) & Chi sq. \\
\hline Mean crime count & $0.059(0.24)$ & $781^{*}$ & $0.060(0.25)$ & $784 *$ \\
\hline $\begin{array}{l}\text { Carry-out liquor } \times \text { on-premises } \\
\text { drinking } \times \text { bus routes }\end{array}$ & $0.185(0.43)$ & $1064 *$ & $0.17(0.42)$ & $984 *$ \\
\hline Level-1 error & $5.98(2.45)$ & - & $5.97(2.44)$ & - \\
\hline
\end{tabular}

Results are based on 33,105 crime locations within 285 census block groups

$* p<.05 ;{ }^{+} p<.08$ 